\title{
¿Qué variables de movilidad están asociadas con la pobreza urbana? El caso de Santiago de Chile y Quito
}

\author{
Susana Herrero Olarte \\ Recibido: 24-06-2020 | Versión final: 04-01-2021
}

Resumen

\begin{abstract}
La pobreza urbana latinoamericana es un ejemplo de segregación residencial. Estudios recientes de tipo cualitativo y cuantitativo respecto a la movilidad y empleo han encontrado una relación positiva entre la distancia al trabajo y el desempleo. El estudio de la movilidad puede ofrecer herramientas fundamentales para la planificación urbana que, de tenerse en cuenta, reducirían la exclusión, el todavía gran desafío. Esta investigación toma las ciudades de Santiago de Chile y Quito para analizar, por barrio, las variables de movilidad para llegar al trabajo que se encuentran relacionadas con la pobreza. Las variables de movilidad consideradas son la distancia desde cada barrio hasta el centro económico y financiero de la ciudad, el coste del transporte para llegar y la densidad del transporte público en cada barrio por persona. El modelo de regresión múltiple de corte transversal aplicado muestra que todas las variables se relacionan con la pobreza, pero que el coste es la que tiene una relación mayor. Dichos hallazgos ofrecen evidencia de que efectivamente el barrio en el que reside una persona está asociado con su nivel de pobreza.
\end{abstract}

Palabras clave: Economía urbana; segregación residencial; acceso; distancia

Citación

\section{Which Mobility Variables are Related to Urban Poverty? The Case of Santiago de Chile and Quito}

Abstract

\begin{abstract}
The Latin American urban poverty constitutes an example of residential segregation. Recent qualitative and quantitative studies on mobility and employment have found a positive relation between distance to work and unemployment. The study of mobility can offer fundamental tools for urban planning that, if are considered, would reduce exclusion, the still great challenge. This research takes the cities of Santiago de Chile and Quito as references for their similarity and availability of data to analyze, by neighborhood, the mobility variables to get to work that are related to poverty. The mobility variables measured in this research are the distance from each neighborhood to the economic and financial center of the city, the cost of transportation to arrive and the density of public transport in each neighborhood per person. The applied crosssection multiple regression model shows that all variables are related to poverty, but the cost is the one with the greater relationship. These findings provide evidence that the neighborhood in which a person resides is associated with his or her level of poverty.
\end{abstract}

Keywords: Urban economy; residential segregation; access; distance

${ }^{1}$ PhD, Universidad de Las Américas, Quito, Ecuador (ORCID ID: 0000-0003-3509-6316, Scopus Author ID: 57194898756, WoS Researcher ID: -2424-2016). Correo de contacto: susana.herrero@udla.edu.ec 


\section{Introducción}

América Latina es la región que más ha aumentado su tasa de urbanización desde 1913 hasta 2008. La falta de planificación urbana ha hecho que el estado de las ciudades latinoamericanas se haya convertido en un límite fundamental para el desarrollo de la región (Herrero Olarte, et al. 2019) Dado que son las nuevas y las pequeñas ciudades las que más crecen es el momento de contar con el mayor número de insumos posible para planificarlas y evitar los problemas de las megalópolis que ya existen.

Entre los retos de las ciudades latinoamericanas a abordar desde su morfología y funcionamiento destaca en especial la pobreza, precisamente por ser un caso de segregación residencial. El barrio en el que una persona reside tipifica su calidad de vida ante sí mismo y ante la sociedad. En el caso de los barrios marginales, queda condicionada su capacidad para desarrollarse pues se limita el acceso a los espacios en los que se concentra el poder económico, político y social.

Para reducir las condiciones de exclusión de los barrios marginados de la periferia de las ciudades, en los que vive el 27\% de la población urbana latinoamericana (NNUU, 2014), es necesario identificar los límites que se generan para producir, y retroalimentar políticas que los minimicen. Entre los límites destaca la movilidad, la misma que hace referencia a los desplazamientos de los individuos, desde una perspectiva que considera su realidad socioeconómica y espacial (Montezuma, 2003).

Cuando las personas que residen en los barrios marginales se desplazan, se enfrentan a algunos problemas, entre los que se encuentran: más tiempo para llegar a su destino, más costos, menor disponibilidad de transporte, y una mayor incomodidad en general; ocasionando que traten de desplazarse lo menos posible. No obstante, movilizarse hacia su sitio de trabajo se vuelve inevitable, especialmente si se considera que la mayor parte de los empleos se concentra en el centro económico de la ciudad; el mismo que, por lo general, les queda lejos. Definir criterios desde la movilidad como herramienta de urbanismo para reducir la exclusión que se genera desde los barrios marginales mejoraría las oportunidades para el desarrollo y reduciría, en consecuencia, la pobreza.

En esta investigación se estudia qué criterios de movilidad se encuentran relacionados con la pobreza de los barrios marginales de la periferia de las ciudades de Santiago de Chile y Quito, tomando como referencia los desplazamientos hacia el centro económico y financiero de cada ciudad.

Las ciudades seleccionadas para el presente estudio son Santiago de Chile y Quito debido a que (i) son ciudades que presentan cierta similitud, y (ii) existe disponibilidad de datos para el cálculo o el uso del IPM (Índice de Pobreza Multidimensional) de cada barrio. Los criterios de movilidad seleccionados fueron la distancia desde cada barrio hasta el centro económico y financiero, el coste del viaje para llegar y la densidad del transporte público por persona. La relación estadística se determinó a través de un modelo de regresión múltiple de corte transversal que incluye las 22 comunas urbanas de Santiago de Chile y las 31 parroquias de la ciudad de Quito.

El resultado del modelo muestra que las tres variables de movilidad consideradas están relacionadas con la pobreza, y que, entre estas, el coste es la que presenta una relación más fuerte. Desde la política pública podría considerase mejorar la movilidad bien subvencionando el transporte colectivo, convertirlo en público o apostar por las centralidades económicas, reduciendo la movilidad.

ACE, 16 (47) CC BY-ND 3.0 ES | UPC Barcelona, España | ¿Qué variables de movilidad están asociadas con la pobreza 


\section{La pobreza urbana en Sudamérica, una cuestión de segregación residencial}

La pobreza urbana en Sudamérica se entiende como el resultado de una estructura política, económica y social que basa su funcionamiento en un sistema que produce una desventaja que se concentra y replica en lo que conocemos como barrios marginales.

Los barrios marginales son homogéneos entre sí. Comparten una dotación insuficiente de los servicios públicos, una distribución de los espacios urbanos inadecuada, un mantenimiento general de la infraestructura pobre y un financiamiento, una gestión y una administración ineficientes. Las viviendas son precarias y en más de la mitad de los casos, ilegales (Durand-Lasservere y Selod, 2009).

La condición común de marginación, compartida entre los barrios sudamericanos, es un fenómeno que en su estudio ha recogido, en la mayoría de los casos a nivel nacional o local, el sentir sobre el desarrollo sudamericano del momento.

Los primeros trabajos sobre la marginación en los barrios llamaban la atención sobre sus causas, que era la migración desde el campo. Los marginados no podían abandonar sus costumbres atrasadas que traían desde el origen (DESAL, 1957) y no estaban preparados naturalmente para adaptarse a la modernidad que suponía la ciudad (Germani, 1980; Medina, 1963). No podían tomar decisiones sobre su realidad inmediata (Giusti, 1973) ni contribuían activamente a la sociedad de la que formaban parte, lo que limitaba su condición de ciudadanos y por ende su capacidad para realizarse como seres humanos (Vekemans y Silva, 1969). Los marginados eran los responsables de su propia condición y debían ser ellos mismos los que se desarrollaran para equiparase al resto de la ciudad.

Para la "teoría de la urbanización dependiente", réplica a pequeña escala de la "teoría de la dependencia”, la marginación era el resultado del modelo capitalista, que explotaba y hacinaba la mano de obra disponible para que no se revelase buscando una mejor calidad de vida (Murmis, 1969). Era necesario, a través de las políticas públicas, generar los mecanismos para impedir que esa "masa marginal" pudiera existir (Nun et al., 1968). Las personas no eran marginadas, sino que estaban en condición de marginación y sólo el Estado podría lograr mejorar su calidad de vida.

Las críticas a la falta de responsabilidad latinoamericana sobre su propia historia y la "teoría de la dependencia”, obligaron a repensar la posición pasiva de los barrios marginales que tenían según la "teoría de la urbanización dependiente", y a reivindicar la responsabilidad individual y comunitaria en el desarrollo. En los barrios marginales las personas podían mejorar de forma autónoma su calidad de vida (De Lomnitz y Fuller, 1975; Pearlman, 1977). Para lograrlo tenían múltiples capacidades, como individuos y como comunidad, que debían poder reconocer y potenciar para alcanzar un verdadero desarrollo (Carrión, 1991).

Desde los noventa se han reconocido, aunque muy matizados, los dos planteamientos que se han ido alternando en el tiempo para definir las estrategias que reduzcan la marginación en los barrios. Por un lado, es necesario reconocer y superar los límites objetivos del contexto; y por el otro, valorar las capacidades de las personas en condición de pobreza para responsabilizarse de su propio desarrollo.

El lugar de nacimiento condiciona las oportunidades (De Barros et al., 2008), el acceso al desarrollo y la libertad (Sen, 1982; Kabeer, 2001). Dado que las personas en condición de marginación nunca estuvieron relacionadas como entes activos en las estructuras política, económica y social (Geremek, 1991) quedan a merced del interés del sistema para mejorar su calidad de vida. Al no alcanzar un mínimo deseado, son evitados por el resto de los grupos sociales. Al limitar su día a día a los barrios,

ACE, 16 (47) CC BY-ND 3.0 ES | UPC Barcelona, España | ¿Qué variables de movilidad están asociadas con la pobreza 
entran en un efecto bucle que alimenta las estructuras que dan lugar a la pobreza crónica (Thang et al., 2006; Ssewanyana, 2009), que afecta a una persona al menos el 50\% de su vida (Carter y Barrett, 2006; Bowles et al., 2006) y que, en términos absolutos, supera en Sudamérica a la pobreza crónica en el campo (Vakis et al., 2016). De superar las condiciones de pobreza, no pasan a formar parte de la clase media, sino que se consideran "vulnerables" por su capacidad para revertir su logro en el corto plazo (Ferreira et al., 2013).

Pese a los límites que presenta el entorno y el contexto, es necesario reconocer las capacidades de los individuos y el grupo para mejorar su calidad de vida, entendidos como "activos", y que siempre pueden mejorarse o potenciarse (Sherraden, 1991), excepto en los peores casos de pobreza crónica y estructural (Carter y Barrett, 2006). Moser et al. (2010) los clasificaban como capital físico, entendido como los recursos que podían generar nuevos recursos; capital financiero disponible, en forma de ahorro o crédito; capital natural, como la tierra, el petróleo, los bosques, los minerales, el agua, etc.; capital humano como el nivel de educación, salud o nutrición; y capital social, entendido como el conocimiento y la pertenencia a la forma de comportamiento generalmente aceptada. Tanto el Banco Mundial, a través del asset vulnerability framework (Moser, 1998) como la CEPAL, mediante el sistema Activos, Vulnerabilidad y Estructura de Oportunidades (AVEO) (Katzman y Filgueira, 1999) proponen metodologías para visibilizarlos y mejorarlos tanto por los afectados, conscientes de su condición de marginación (Sabatini et al., 2001) como desde intervenciones externas para el desarrollo.

Por su capacidad cuantitativa para identificar los límites objetivos para el desarrollo de los barrios marginales, así como los "activos" disponibles, la movilidad urbana, se convierte en una herramienta clave para reducir la pobreza en las ciudades, máxime cuando esta es una cuestión de segregación residencial, considerando que esta última denota la existencia de una distribución desigual de los grupos socioeconómicos en las ciudades (Rodríguez, 2008).

\section{Movilidad urbana como factor de pobreza}

La movilidad estudia las formas de desplazamiento, su contexto, sus causas y sus consecuencias (Miralles-Guasch, 2002; Sanz, 2005). Como se mencionó anteriormente, la movilidad urbana es un concepto que permite entender los desplazamientos de los individuos desde una perspectiva que considera su situación socioeconómica y su entorno en general (Montezuma, 2003). Según lo abordado por el autor, este concepto es más amplio que el de transporte o desplazamientos pues permite centrar el análisis en la persona y su entorno. En Latinoamérica es el resultado del análisis coste-beneficio de los procesos de aglomeración (Rosenthal y Strange, 2004), esto es, cómo y porqué las ventajas de la acumulación están condicionadas por los costes de congestión (Henderson, 2002; Timmins y Murdock, 2007).

El trabajo en torno a la movilidad tiene además como vocación el contribuir a la planificación urbana de las ciudades de menos de un millón de habitantes, que se espera sea el grupo que más aumente no sólo en cantidad sino la población (NNUU, 2014), y que han ocupado hasta ahora un papel secundario en la planificación por la tradición centralista de los presupuestos latinoamericanos. Los retos que se generan al abordar la problemática de movilidad urbana son algunos, entre los más representativos está la discusión de un transporte público de mayor calidad (mejor relación tiempo de viaje - costo), seguridad vial, emisiones de contaminantes, niveles de congestión de tránsito, y la gestión de tránsito en general (Thorson Jorgensen, 2012).

El análisis de movilidad está además condicionado por la heterogeneidad de la densidad urbana entre los grupos económicos. El estudio realizado en las ciudades de Santiago de Chile y Quito, recogido en la Figura 1, muestra cómo las clases altas están concentradas en pequeños epicentros económicos

ACE, 16 (47) CC BY-ND 3.0 ES | UPC Barcelona, España | ¿Qué variables de movilidad están asociadas con la pobreza 
en la ciudad y dispersas en la periferia, mientras los grupos más desfavorecidos, que ocupan la mayor extensión territorial, presentan mayores niveles de concentración territorial. Las clases medias presentan bajas tasas de densidad. Mayor densificación, sobre todo en los estratos medios, generaría una mejora de la oferta del transporte colectivo, público y privado, presentándose como alternativa real al automóvil para los trayectos diarios.

Figura 1. Densidad y población de Santiago de Chile y Quito por grupos de barrios de pobreza

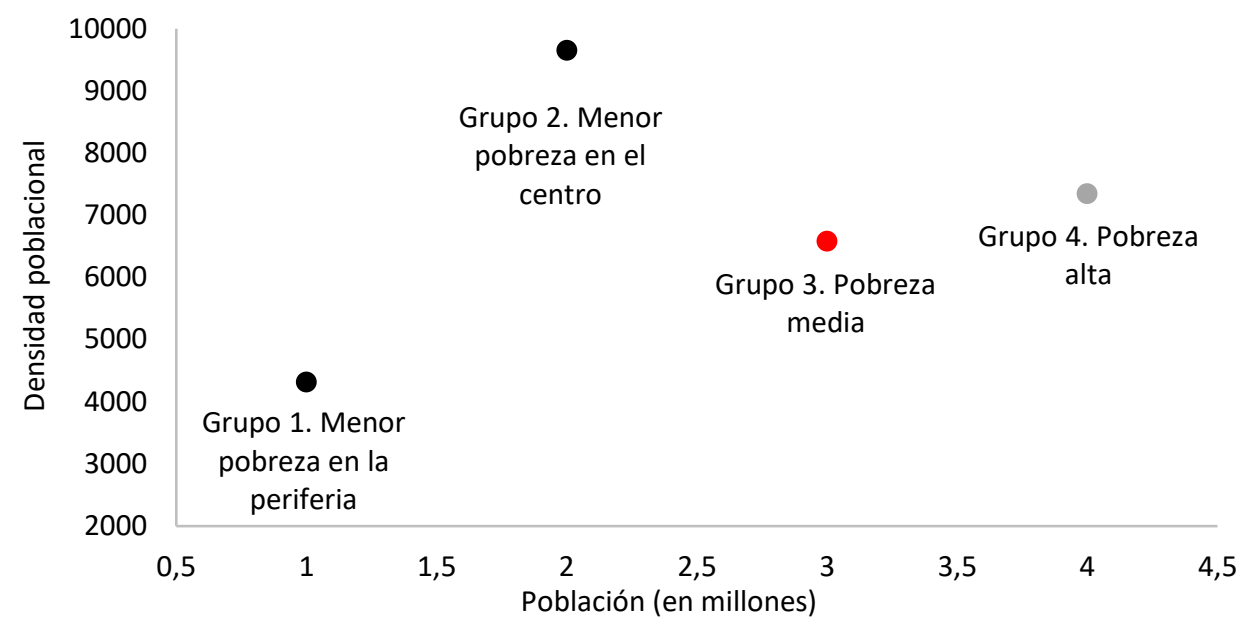

Fuente: Elaboración propia en base a NNUU, 2014

Al reto de la heterogeneidad en la ciudad se le añade el de una perspectiva de la densidad futura a la baja. Desde 1990 y hasta 2015 la superficie construida en las ciudades latinoamericanas es mayor todos los años al crecimiento de la población. El aumento de la superficie urbana se explica por las nuevas construcciones en las ciudades que ya existen, y por la aparición de otras nuevas ciudades, con una infraestructura de transporte colectivo que trata de adaptarse a una morfología que se da de manera natural sin un diseño urbano previo. En Europa y los EEUU el crecimiento se explica por la anexión de pueblos colindantes o espacios verdes aledaños, lo que minimiza el impacto en términos de movilidad, y no por un déficit habitacional. El grupo de edad que necesita su primera vivienda, de entre 20 y 29 años, supone el 17\% de la población latinoamericana, frente al 14\% en los EEUU y el 12\% en Europa. Son 107 millones de latinoamericanos, 80 de estadounidenses y 50 de europeos. La brecha aumenta al considerar el grupo de entre 10 y 19 años (NNUU, 2015).

La relación entre los costes y los beneficios de la aglomeración debe regularse necesariamente desde el sector público. En Latinoamérica, las ciudades han evolucionado en un contexto de intervención pública que no ha logrado minimizar una congestión que supone, para la cuarta parte de la ciudadanía, tardar al menos una hora en llegar al trabajo por la saturación de las vías disponibles en unas condiciones, para el $25 \%$ de los que van en transporte colectivo, deficientes o muy deficientes (CAF, 2017). El reto inmediato es mejorar la infraestructura y el sistema colectivo de transporte.

La infraestructura es insuficiente porque no ha habido durante años la inversión necesaria (CG/LA, 2017) que garantice un promedio comparable con el promedio de la OCDE y homogenice los datos, sobre todo entre las capitales y el resto de las ciudades. El 90\% de las ciudades tiene menos de 30 km de vía por cada 10.000 habitantes, mientras en las 50 ciudades económicamente más importantes de los EEUU el promedio oscila entre los 51 y los $71 \mathrm{~km}$ (Zhao y Levinson, 2012). El número de semáforos varía entre los tres y los 34 por cada $100 \mathrm{~km}$. En algunas ciudades, y de manera excepcional, se registra un máximo del 9\% de vías con prioridad para bicicletas y del 3\% para el

ACE, 16 (47) CC BY-ND 3.0 ES | UPC Barcelona, España | ¿Qué variables de movilidad están asociadas con la pobreza urbana? El caso de Santiago de Chile y Quito. DOI: http://dx.doi.org/10.5821/ace.16.47.9510 
transporte público. En todos los casos los datos son inferiores en las pequeñas y medianas ciudades. Se presenta además una deficiencia clara en los medios públicos de transporte, que no llegan al 20\% de los ciudadanos latinoamericanos (CAF, 2016). El déficit público lo cubre el sistema privado de transporte colectivo, con unos requisitos en la calidad del servicio supeditados a la optimización de sus beneficios.

El 39\% de los viajes se realizan en transporte colectivo, público o privado, el $26 \%$ a pie y el $22 \%$ en automóvil (CAF, 2017). Pese a su menor uso, el automóvil genera el 85\% del CO2 en las ciudades, el $35 \%$ de las muertes en la vía -frente al 0,7 en transporte colectivo- y supone el 65\% del coste total del transporte no comercial (OMU y CAF, 2010). A pesar de sus desventajas comparadas, se espera que su uso sea el que más aumente, en un 14\%, de 2015 a 2020 (Fulton y Eads, 2004).

Las desventajas del automóvil quedan superadas por sus ventajas. El trayecto en automóvil supone un ahorro del 65\% del tiempo (CAF, 2017), permite ignorar las incomodidades de los medios de transporte público (Owens, 1996), posibilita seguir el paradigma de la ciudad jardín de Howard manteniendo el acceso a las oportunidades laborales y sociales de la ciudad e implica el pertenecer a una mínima clase económica (Whitelegg, 1997).

El transporte colectivo es, por lo tanto, la expresión física de la exclusión por movilidad. Son las clases más bajas las que utilizan el transporte colectivo (Gomide, 2003) porque supone un coste anual tres veces menor respecto al privado (OMU y CAF, 2010). Resulta, en términos relativos, más caro para los grupos más desfavorecidos, a los que les suponía el transporte el 16\% de su ingreso frente al 5\% para el estrato más rico en Colombia (Observatorio de Bogotá, 2008). Por tanto, tienen que seleccionar muy bien cuándo y para qué se desplazan (Allport, 2000). En términos absolutos se movilizarán menos que el resto de los grupos sociales, reduciendo el acceso a las oportunidades que puede ofrecer la ciudad (Companhia do Metropolitano de Sao Paulo, 1998) y, por lo tanto, el ejercicio comparado de sus derechos (Tolley y Turton, 2014). Además del coste, la disponibilidad del transporte limita su uso, como en los asentamientos ilegales, donde el 15\% de la ciudadanía no tiene acceso a ningún sistema de transporte, público o privado.

La movilidad se convierte así en causa y consecuencia de los procesos de marginación generadores de pobreza. Mejorar las condiciones de movilidad para mejorar el acceso a las oportunidades en los barrios marginados permitiría reducir la exclusión y, en consecuencia, la pobreza (ITRANS, 2004; Figueroa, 2005). Aquí resalta la importancia de entender y verificar la relación estadística entre ambas variables (movilidad urbana y pobreza), con el fin de poder orientar de mejor forma políticas públicas que mejoren las oportunidades de aquellos segmentos de la sociedad que han sido normalmente excluidos o perjudicados.

El acceso al empleo destaca entre las distintas dimensiones que podrían tratarse a través de la mejora de la movilidad por su relación con la pobreza. En los trabajos que relacionan movilidad y empleo, los resultados cuantitativos y cualitativos muestran que existe una relación indirecta entre distancia y calidad del empleo, y una relación directa con el desempleo (Sabatini et al., 2001; Borsdorf, Bähr y Janoschka, 2002).

Esta investigación trata de complementar el trabajo, expuesto anteriormente, que relaciona la movilidad desde los barrios marginados con el empleo, con un doble propósito: (i) estudiar si la movilidad se relaciona significativamente con la pobreza en los barrios marginales; y (ii) qué variables de movilidad presentan una relación más fuerte. El estudio se realiza tomando como muestra las ciudades de Santiago de Chile y Quito, por las razones previamente presentadas en la introducción.

ACE, 16 (47) CC BY-ND 3.0 ES | UPC Barcelona, España | ¿Qué variables de movilidad están asociadas con la pobreza 


\section{Metodología}

El presente estudio pretende relacionar estadísticamente la pobreza y la movilidad en los barrios de Santiago de Chile y Quito. La variable dependiente es el índice de pobreza multidimensional IPM y las variables independientes de movilidad son: la distancia de la localidad de estudio hacia el centro financiero, el coste del viaje y la densidad del transporte público por persona. Para poder validar el modelo, como variables de control se incluyeron la etnia y el sexo. Estas dos variables de control fueron seleccionadas pues las mismas son importantes para poder explicar los niveles de pobreza presentes en las distintas parroquias/comunas incluidas en el estudio.

Por un lado, se espera que, siempre y cuando las mujeres logren ingresar al mercado laboral, la pobreza reduzca ante mayor presencia de mujeres considerando que puede haber una fuente adicional de ingresos en los hogares (Parada, 2016), además, se considera que las mujeres pueden ser mejores administradoras de recursos, permitiendo así disminuir los niveles de pobreza (Valencia, 2008). Por otro lado, la composición étnica de las parroquias resulta relevante como un control debido a la exclusión social étnica que existe en los países de la región (Flórez y Medina, 2003), y que se manifiesta en la participación y resultados negativos en el mercado laboral (Meneses, et al., 2021). Por esta razón, se espera que una mayor composición indígena de la parroquia, este asociado con mayores niveles de pobreza.

La relación estadística trata de determinarse a través de un modelo de regresión múltiple de corte transversal que incluye las 22 comunas urbanas de Santiago de Chile y las 31 parroquias de la ciudad de Quito1. Se presenta a continuación el modelo en su forma específica (1):

$\log I P M=\alpha+\beta \log$ Distancia $+\delta \log$ Costo $+\gamma \log$ Densidadpercapita + OlogIndigenas $+\varphi$ logMujeres $+\varepsilon_{t}$

Donde IPM es el índice de pobreza multidimensional; distancia es la distancia entre cada parroquia/comuna y la parroquia/comuna que concentra la mayor cantidad de empleos en la ciudad; costo es el coste en transporte público en dólares para llegar entre parroquias; densidadpercapita es la densidad de transporte público en cada parroquia, medida a partir de la frecuencia de transporte público en la parroquia en relación al número de habitantes de la misma; indígenas es el porcentaje de población indígena en cada parroquia y mujeres el porcentaje de mujeres por parroquia.

Para calcular el IPM se toma la metodología propuesta de Alkire y Foster (2007), referente en el área. Emplean las dimensiones de educación, salud, trabajo y seguridad social, y vivienda y entorno. La metodología parte con la selección de dimensiones, sobre las cuales se obtienen ciertos indicadores; a partir de esto, se define la unidad de análisis e identificación; el siguiente paso corresponde a las etapas de agregación; seguido por la definición de una estructura de ponderación; y, finalmente, la selección del umbral para la definición de pobreza multidimensional. Para el cálculo en Quito a nivel de parroquia se utilizaron los datos del Censo de Población y Vivienda de 2010 (INEC, 2011). A partir del Censo se pueden calcular diez de los doce indicadores que emplean Alkire y Foster para el cálculo del IPM, lo que no resulta un problema al utilizarse la misma sintaxis. Los datos por comuna de la Región Metropolitana de Santiago fueron obtenidos de la Encuesta de Caracterización Socioeconómica Nacional, Gajardo (2016), publicada por el Ministerio de Desarrollo Social. Aquí sí pudieron utilizarse los doce indicadores de referencia.

\footnotetext{
1 Excluido el centro financiero: Belisario Quevedo, Carcelén, Centro Histórico, Chilibulo, Chillogallo, Chimbacalle, Cochapamba, Comité Del Pueblo, La Concepción, Cotocollao, El Condado, Guamaní, Itchimbía, Jipijapa, Kennedy, La Argelia, La Ecuatoriana, La Ferroviaria, La Libertad, La Magdalena, La Mena, Mariscal Sucre, Ponceano, Puengasí, Quitumbe, Rumipamba, San Bartolo, San Isidro Del Inca, San Juan, Solanda, Turubamba.
}

ACE, 16 (47) CC BY-ND 3.0 ES | UPC Barcelona, España | ¿Qué variables de movilidad están asociadas con la pobreza 7 urbana? El caso de Santiago de Chile y Quito. DOI: http://dx.doi.org/10.5821/ace.16.47.9510 
Las variables de desplazamiento toman como referencia física la parroquia con una mayor capacidad para generar empleo. En Quito, la información está proporcionada por el Municipio de Quito, en base al análisis de una muestra de 347 empresas de las casi 3.600 activas con sede en la ciudad en 2010. La parroquia que genera más empleo, con casi el 23\% del total, es Iñaquito. En el caso de la ciudad de Santiago, se tomaron datos de los Reportes Estadísticos Comunales (2015) de la Biblioteca del Congreso Nacional de Chile. La comuna que más empleos genera es Providencia.

Para seleccionar las variables de interés relacionadas con movilidad, se toma en consideración los resultados de las encuestas a los usuarios del transporte público de la CAF (2017) en ocho grandes ciudades latinoamericanas, y del BID (2015), en 30 ciudades pequeñas y medianas. En las ciudades grandes los principales problemas son el tiempo del trayecto con el 18,3\%, la escasa frecuencia con el 15,3\%, la seguridad dentro del vehículo y el confort que suman el 24\%, y al coste con el 9,3\%. Le siguen la seguridad vial, la confiabilidad del horario y la seguridad en la parada. En las ciudades pequeñas y medianas, el $78 \%$ de los entrevistados considera que el transporte público es incómodo y está sucio, el $74 \%$ está disconforme con la frecuencia y el 56\% considera que el precio es alto o muy alto.

De entre las variables de movilidad identificadas por los usuarios se han seleccionado para esta investigación el tiempo, la frecuencia, que se entenderá como la densidad del transporte, pues se espera que exista una relación positiva entre la densidad poblacional y la frecuencia del servicio de transporte público en la parroquia (Salazar-Burrows, et al., 2014), y el coste. La seguridad dentro del vehículo y el confort no se han considerado dentro del estudio pues existe poca variabilidad de estas entre los distintos barrios de cada una de las ciudades considerados en el análisis.

La variable distancia está medida en metros, y la selección de la ruta para su medición, se realizó en base a la vía más rápida desde el centro de cada localidad hacia el centro de la localidad generadora de empleo; Iñaquito para la ciudad de Quito y Providencia para la ciudad de Santiago. La medición se realizó con la herramienta Google Maps, que realiza estimaciones satelitales en tiempo real de las rutas, las distancias y las frecuencias del transporte público de las localidades de estudio.

La variable coste se define como el valor monetario que las personas deben pagar para movilizarse hacia el centro financiero haciendo uso del transporte público. Teniendo en cuenta la distancia, y de acuerdo con el número de trasbordos de cada localidad hasta el centro financiero, se calcularon los costos por viaje; suponiendo que cada trasbordo implica un coste adicional. Se utilizaron los tipos de cambio del Banco Central del Ecuador para obtener, también en Chile, valores en dólares estadounidenses. Los precios por viaje se tomaron de las agencias de transporte público oficiales de cada ciudad en 2018. En Quito de la Empresa Publica Metropolitana de Transporte de Pasajeros y en Santiago del Directorio de Transporte Público Metropolitano. El costo por viaje en Quito es de USD 0.25 en todos los horarios y en Santiago es de 660 pesos chilenos o USD 1,04 de acuerdo con la tarifa Valle. Incluye Metro, Metrotren y Bus en los horarios comprenden de lunes a viernes, entre las 06:30 a 06:59 horas, de 09:00 a 17:59 horas, o de 20:00 a las 20:44 horas (Directorio de Transporte Público Metropolitano, 2017).

La variable densidad del transporte público per cápita es la cantidad de veces que los buses pasan por la parada inicial de cada localidad en el transcurso de un día con relación al número de habitantes de cada localidad. La aplicación Google Maps estima la frecuencia en la que una unidad de transporte pasa por una determinada parada durante un intervalo de tiempo -cada tres minutos, cinco minutos, etc. Se sumaron las frecuencias de todas las rutas posibles y se realizó una transformación matemática para estandarizarla en intervalos diarios. De acuerdo con esto, se midió la frecuencia para cada parroquia o comuna con observaciones diarias durante una semana en intervalos de la

ACE, 16 (47) CC BY-ND 3.0 ES | UPC Barcelona, España | ¿Qué variables de movilidad están asociadas con la pobreza 
mañana, del medio día y de la tarde. En total se obtuvieron 688 observaciones, de las que se calcularon las medianas para incluirlas en el modelo.

Una de las limitaciones que presenta la metodología planteada es que a partir de la misma no se puede hacer inferencia causal entre las variables relacionadas a movilidad y la pobreza en los barrios de Santiago de Chile y Quito, pues para esto, sería necesario considerar en el modelo potenciales problemas de endogeneidad, y plantear una metodología que permita realizar esta inferencia. Por esta razón, los resultados que se presentan sólo deben ser considerados como asociaciones o correlaciones entre las variables de interés.

\section{Resultados}

La Tabla 1 muestra la relación estadística entre el índice de pobreza multidimensional (IPM) y las variables distancia de la localidad de estudio hacia el centro financiero, coste del viaje y densidad del transporte público por persona, a través de un modelo de regresión múltiple de corte transversal para las 31 parroquias ${ }^{2}$ y 22 comunas $^{3}$ de Quito y Santiago, respectivamente.

Tabla 1. Modelo de regresión múltiple log-log

\begin{tabular}{|c|c|}
\hline & Índice de Pobreza Multidimensional \\
\hline \multirow[t]{2}{*}{ Log Distancia } & $0.226 \star \star \star$ \\
\hline & $(0.101)$ \\
\hline \multirow[t]{2}{*}{ Log Costo } & 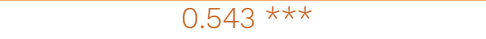 \\
\hline & $(0.099)$ \\
\hline \multirow[t]{2}{*}{ Log Densidad } & 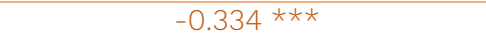 \\
\hline & $(0.114)$ \\
\hline \multirow[t]{2}{*}{ Log Indigena } & $0.479 * \star \star$ \\
\hline & $(0.080)$ \\
\hline \multirow[t]{2}{*}{ Log Mujer } & $-0.850 * \star \star \star$ \\
\hline & $(0.181)$ \\
\hline \multirow[t]{2}{*}{ _cons } & 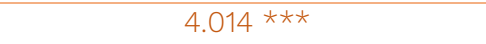 \\
\hline & (1.833) \\
\hline Obs & 53 \\
\hline$F(3,27)$ & 41.23 \\
\hline Prob $>F$ & 0.0000 \\
\hline R-squared & 0.8143 \\
\hline Adj R-squared & 0.7946 \\
\hline
\end{tabular}

pvalor * $0.1 * \star 0.05 * * * 0.01$

Fuente: Elaboración propia

El coeficiente de bondad de ajuste del modelo es del 0.81 y todas las variables tienen un valor $p$ menor a 0,05, lo que muestra que todas las variables son significativas para explicar la pobreza y que existe significancia global en el modelo.

2 Excluido el centro financiero: Belisario Quevedo, Carcelén, Centro Histórico, Chilibulo, Chillogallo, Chimbacalle, Cochapamba, Comité Del Pueblo, La Concepción, Cotocollao, El Condado, Guamaní, Itchimbía, Jipijapa, Kennedy, La Argelia, La Ecuatoriana, La Ferroviaria, La Libertad, La Magdalena, La Mena, Mariscal Sucre, Ponceano, Puengasí, Quitumbe, Rumipamba, San Bartolo, San Isidro Del Inca, San Juan, Solanda, Turubamba.

${ }^{3}$ Excluido el centro financiero: Santiago, Cerro Navia, Conchalí, El Bosque, Estación Central, La Florida, La Granja, La Pintana, Las Condes, Macul, Maipú, Ñuñoa, Pedro Aguirre Cerda, Peñalolén, Pudahuel, Quilicura, Quinta Normal, Recoleta, Renca, San Miguel, Puente Alto, San Bernardo

ACE, 16 (47) CC BY-ND 3.0 ES | UPC Barcelona, España | ¿Qué variables de movilidad están asociadas con la pobreza urbana? El caso de Santiago de Chile y Quito. DOI: http://dx.doi.org/10.5821/ace.16.47.9510 
Los valores de los regresores son diferentes de cero y los signos son los esperados por la teoría. Una mayor distancia y un mayor costo incrementan la pobreza mientras que una mayor densidad de transporte público per cápita la disminuye, esto último es esperable considerando la forma en la que se define a la densidad de transporte público en el presente estudio, medida que considera la frecuencia de las unidades de transporte en una determinada parroquia, respecto al total de la población en dicha parroquia. Por tanto, un incremento en dicha densidad puede representar mayor acceso de la población de la parroquia a los servicios de transporte público, lo cual está asociado de forma indirecta con los niveles de pobreza. El signo de las variables de control es igualmente el esperado. Por un lado, se espera que a medida que el número de personas auto identificadas como indígenas aumenta, los niveles de pobreza en dicha parroquia también aumenten, esto como se mencionó anteriormente, está asociado a la exclusión social étnica que presentan estos grupos, especialmente en el mercado laboral. Por otro lado, el signo negativo asociado a la proporción de mujeres en la parroquia se explica debido a que, como ha sido discutido en la literatura, las mujeres tienden a dar un mejor uso de los recursos, lo cual, indudablemente puede ayudar a disminuir los niveles de pobreza de una determinada zona.

El factor de inflación de la varianza es menor a diez en todas las variables, por lo que se resuelve que no existe multicolinealidad en ninguna de las variables. La prueba de normalidad de los residuos se realiza mediante los tests Jarque-Bera y Shapiro-Wilk. Los resultados de las pruebas realizadas indican que los residuos siguen una distribución de probabilidad normal, tanto en Jarque-Bera como Shapiro-Wilk, y los valores p son mayores a 0,05 por lo que se acepta la hipótesis de normalidad. La última prueba que se aplica al modelo es para constatar si las varianzas de las perturbaciones no son constantes, para lo cual se procede con la prueba Breusch-Pagan. El valor p resultante de la prueba de Breusch-Pagan es mayor que 0,05, por lo que se acepta la hipótesis de que las varianzas de las perturbaciones son constantes a lo largo de las observaciones, lo que quiere decir que no existen problemas de heterocedasticidad en el modelo planteado.

\section{Conclusiones}

La segregación residencial en las ciudades de Quito y Santiago de Chile es capaz de mantener la pobreza en los espacios físicos tradicionalmente marginados. El análisis realizado en estas ciudades muestra que el barrio en el que una persona reside está relacionado con su pobreza. El estudio revela la relación existente entre la distancia desde la parroquia/comuna de residencia hasta la parroquia/comuna que genera más empleos en cada una de las dos ciudades, y las limitaciones de acceso al mercado laboral.

El modelo utilizado muestra que las variables consideradas, esto es, la distancia entre las dos parroquias, la densidad del transporte para llegar y el coste en medios públicos, se relacionan de forma significativa con la pobreza en las dos ciudades. El signo es además el esperado según la revisión de literatura planteada. La pobreza se relaciona positivamente con la distancia y con el coste para llegar de la una a la otra; y negativamente con la densidad de los medios de transporte. Las variables de control, tradicionalmente vinculadas con la pobreza, se relacionan según lo esperado. De las tres variables consideradas el coste es la que tiene un mayor peso.

En este contexto, surge a la luz de los principales resultados la necesidad de plantear nuevos escenarios que consideraren políticas dirigidas a mejorar el acceso la parroquia/comuna con la mayor capacidad para generar empleo, valorando especialmente el abaratamiento de los costes; o que posicionen nuevas centralidades que alivien la presión sobre un único centro económico.

ACE, 16 (47) CC BY-ND 3.0 ES | UPC Barcelona, España | ¿Qué variables de movilidad están asociadas con la pobreza 
Conflicto de intereses: La autora declara que no hay conflicto de intereses.

\section{Bibliografía}

Alkire, S. y Foster, J. (2007). Counting and multidimensional poverty measurement. Journal of Public Economics, 95(2011), 476-487. DOI: https://doi.org/10.1016/j.jpubeco.2010.11.006

Allport, R. (2000). Transport services for the urban poor. Infrastructure for Development: Private Solutions and the Poor. Trabajo presentado en la Conferencia Internacional de Infraestructura para el desarrollo: Soluciones Privadas y Pobreza, Londres, Inglaterra.

BID. (2015). Trasporte: Mejorar la calidad de vida mediante proyectos de infraestructura y transporte. Recuperado de https://www.iadb.org/es/sectores/transporte/perspectiva-general

Borsdorf, A.; Bähr, J. y Janoschka, M. (2002). Die Dynamik stadtstrukturellen Wandels in Lateinamerika im Modell der lateinamericanischen Stadt. Geographica Helvetica, 57(4), 300-310. DOI: https://doi.org/10.5194/gh-57-300-2002

Bowles, S. ; Durlauf, S., y Hoff, K. (2006). Poverty traps. New Jersey, Estados Unidos: Princeton University Press.

CAF (2016). Observatorio de Movilidad Urbana: Informe 2015-2016. Recuperado de http://scioteca.caf.com/handle/123456789/981

CAF. (2017). Crecimiento urbano y acceso a oportunidades: un desafío para América Latina. Bogotá, Colombia: Panamericana Formas e Impresos S.A.

Carrión, F. (1991). La Investigación Urbana en América Latina. Caminos Recorridos y por Recorrer. Quito, Ecuador: CIUDAD.

Carter, M. y Barrett, C. (2006). The economics of poverty traps and persistent poverty: An asset-based approach. The Journal of Development Studies, 42(2), 178-199. DOI: https://doi.org/10.1080/00220380500405261

CG/LA (2017). Infraestrucutre. Recuperado de https://www.cg-la.com/

Companhia do Metropolitano de Sao Paulo. (1998). Pesquisa Origem-destino 1997. Recuperado de https://transparencia.metrosp.com.br/dataset/pesquisa-origem-e-destino

De Barros, R.; Vega, J. y Saavedra, J. (2008). Measuring inequality of opportunities for children. Washington DC, Estados Unidos: The World Bank.

De Lomnitz, A. y Fuller, L. (1975). Como sobreviven los marginados. México DF, México: Siglo Veintiuno Editores.

DESAL. (1957). Una estrategia contra la miseria. Santiago de Chile, Chile: DESAL.

Directorio de Transporte Público Metropolitano. (2017). Tarifas Vigentes. Recuperado de https://www.dtpm.cl/index.php/2013-04-24-14-09-34/2013-04-26-16-56-48

Durand-Lasserve, A. y Selod, H. (2009). The formalization of urban land tenure in developing countries. En Lall S.V.; Freire M.; Yuen B.; Rajack R. y Helluin JJ. (eds). Urban Land Markets, (pp. 101-132). DOI: https://doi.org/10.1007/978-1-4020-8862-9 5

ACE, 16 (4.7) CC BY-ND 3.0 ES | UPC Barcelona, España | ¿Qué variables de movilidad están asociadas con la pobreza urbana? El caso de Santiago de Chile y Quito. DOI: http://dx.doi.org/10.5821/ace.16.47.9510 
Directorio de Transporte Público Metropolitano. (2017). Pasajeros por tarifas 2017. Recuperado de http://www.trolebus.gob.ec/index.php/gestion/indicadores/indicadores-operacionales2017/pasajeros-por-tarifas-2017

Ferreira, F.; Messina, J.; Rigolini, J.; López-Calva, L.; Lugo, M. y Vakis, R. (2013). Economic Mobility and the Rise of the Latin American Middle Class. Washington DC, Estados Unidos: World Bank.

Figueroa, O. (2005). Transporte urbano y globalización. Políticas y efectos en América Latina. EURE. 94(1). 41-53. DOI: https://doi.org/10.4067/s0250-71612005009400003

Flórez, C. E.; Medina, C. y Urrea, F. (2003). Los costos de la exclusión social por raza o etnia en América Latina y el Caribe. Coyuntura Social. 29(1). 46-69. Recuperado de https://www.repository.fedesarrollo.org.co/handle/11445/1061

Fulton, L. y Eads, G. (2004). Model Documentation and Reference Case Projection. IEA/SMP, 1(2). 196. Recuperado de https://www.eea.europa.eu/data-and-maps/data-providers-and-partners/httpwww.wbcsd.org-web-publications-mobility.pdf

Gajardo, S. (2016). Pobreza y distribución del ingreso en la Región Metropolitana de Santiago: resultados encuesta casen 2015. Seremi de Desarrollo Social Metropolitana. 3(2). 4-20 Recuperado de https://www.gobiernosantiago.cl/wp-content/uploads/2014/12/DOCUMENTO-POBREZA-Y-DISTRING-RMS-CASEN-2015.pdf

Geremek, B. (1991). Les fils de Caïn. París, Francia: Flammarion.

Germani, G. (1980). El concepto de marginalidad: significado, raíces históricas, y cuestiones teóricas, con particular referencia a la marginalidad urbana. Buenos Aires, Argentina: Ediciones Nueva Visión.

Giusti, J. (1973). Organización y participación popular en Chile: el mito del hombre marginal. Buenos Aires, Argentina: Ediciones Flacso.

Gomide, A. (2003). Transporte urbano e inclusão social: elementos para políticas públicas. Instituto de Pesquisa Economica Aplicada. 960(1), 7-31. Recuperado de http://repositorio.ipea.gov.br/handle/11058/2893

Henderson, V. (2002). Urban primacy, external costs, and quality of life. Resource and Energy Economics, 24(1-2), 95-106. DOI: https://doi.org/10.1016/S0928-7655(01)00052-5

INEC. (2011). Censo de Población y Vivienda 2010. Recuperado de http://www.ecuadorencifras.gob.ec/base-de-datos-censo-de-poblacion-y-vivienda-2010/

ITRANS. (2004). Mobilidade e pobreza Pesquisa sobre a mobilidade urbana na populacao de baixa renda. Relatorio final. Brasilia, Brasil: Instituto de Desenvolvimento e Informação em Transporte.

Kabeer, N. (2001). Conflicts over credit: re-evaluating the empowerment potential of loans to women in rural Bangladesh. World development, 29(1), 63-84. DOI: https://doi.org/10.1016/S0305$\underline{750 \times(00) 00081-4}$

Kaztman, R. y Filgueira, C. (1999). Marco conceptual sobre activos, vulnerabilidad y estructura de oportunidades. Comisión Económica para América Latina y el Caribe CEPAL, 176(1). 7-26. Recuperado de https://www.cepal.org/es/publicaciones/28663-marco-conceptual-activos-vulnerabilidadestructuras-oportunidades

Medina, J. (1963). El desarrollo social de América Latina en la postguerra. Buenos Aires, Argentina: Solar/ Hachette.

Meneses, K.; Córdova Montero, G. y Aguirre Soria, K. (2021). Lo más destacado y sobresaliente que caracteriza al mercado laboral ecuatoriano en siete hechos estilizados. Revista Economía Y Política, (33), 1-33. Recuperado de https://publicaciones.ucuenca.edu.ec/ojs/index.php/REP/article/view/3482

ACE, 16 (47) CC BY-ND 3.0 ES | UPC Barcelona, España | ¿Qué variables de movilidad están asociadas con la pobreza 12 urbana? El caso de Santiago de Chile y Quito. DOI: http://dx.doi.org/10.5821/ace.16.47.9510 
Miralles-Guasch, C. (2002). Transporte y territorio urbano. Documents d'analisi geografica, 41(1), 107120. Recuperado de https://raco.cat/index.php/DocumentsAnalisi/article/view/31773.

Montezuma, R. (2003). Ciudad y transporte: la movilidad urbana. En Osmont A.; Balbo M.; Borja J.; Durand-Lasserve A.; Carrión, F.; Burgess, R.; Petrella, L y Vanderschueren, F.; Levy C.; Simioni, D. (eds). La Ciudad Inclusiva. (pp. 175-192). Santiago de Chile, Chile: CEPAL.

Moser, C., Norton, A., Stein, A. y Georgieva, S. (2010). Pro-Poor Adaptation to Climate Change in Urban Centers. World Bank Publications. 54947(1). 1-69. Recuperado de https://openknowledge.worldbank.org/handle/10986/3001

Moser, C.O. (1998). The asset vulnerability framework: reassessing urban poverty reduction strategies. World development, 26(1), 1-19. DOI: https://doi.org/10.1016/S0305-750X(97)10015-8

Murmis, M. (1969). Tipos de marginalidad y posición en el proceso productivo. Revista Latinoamericana de Sociología. 5(2), 413-421. Recuperado de http://www.scielo.org.co/scielo.php?script=sci nlinks\&re $\mathrm{f}=000120 \& \mathrm{pid}=\mathrm{S} 0120-4807201200020000200010 \& \ln g=e n$

NNUU. (2015). World Population Prospects: The 2015 Revision, Key Findings and Advance Tables. Recuperado de https://population.un.org/wpp/Publications/Files/Key Findings WPP 2015.pdf

NNUU. (2014). World Urbanization Prospects, the 2014 revision. Recuperado de https://esa.un.org/unpd/wup/

Nun, J., Marín, J.C. y Murmis, M. (1968). La marginalidad en América Latina. Informe preliminar. Instituto Torcuato Di Tella, Centro de Investigaciones Sociales. 53(1), 3-79. Recuperado de https://hum.unne.edu.ar/biblioteca/apuntes/Apuntes\%20Ciencias\%20de\%20la\%20Educacion/Socio logia/Unidad5/Marginalidad\%20en\%20america\%20latina.pdf

Herrero Olarte, S. H.; Villa, A. y Sandoval, S. (2019). ¿Influye la diversidad económica del barrio en su tasa de pobreza? El caso de Quito. ACE: Architecture, City and Environment = Arquitectura, Ciudad y Entorno, 13 (39): 145-160. DOI: http://dx.doi.org/10.5821/ace.13.39.5661

Observatorio de Bogotá. (2008). Caracterización e indicadores de la movilidad en Bogotá; 2007. Bogotá, Colombia: Cámara de Comercio de Bogotá.

OMU y CAF. (2010). Observatorio de Movilidad Urbana. CAF. Recuperado de http://omu.caf.com

Owens, S. (1996). I wouldn't start from here: land use, transport and sustainability. En Cartledge B., Chase R., Dower M., Goodwin P., Morton A., Reid B., Somerville H. Transport and the Environment, (pp. 45-61). Oxford, Inglaterra: Oxford Univ. Press 1996

Parada, C. (2016). Empleo femenino, pobreza y desigualdad. Un análisis de microdescomposiciones Uruguay (1991-2012). El trimestre económico, 83(330), 371-404. Recuperado de http://www.scielo.org.mx/scielo.php?script=sci arttext\&pid=S2448-718X2016000200371\&lng=es\&tlng=es

Pearlman, J. (1977). O Mito da Marginalidade: favelas e política no Rio de Janeiro. Rio de Janeiro, Brasil: Paz e Terra.

Rodríguez, J. (2008). Movilidad cotidiana, desigualdad social y segregación residencial en cuatro metrópolis de América Latina. EURE (Santiago). 34(103), 49-71. DOI: https://dx.doi.org/10.4067/S0250$\underline{71612008000300003}$

Rosenthal, S. y Strange, W. (2004). Evidence on the nature and sources of agglomeration economies. Handbook of Regional and Urban Economics. 4(1), 2119-2171. DOI: https://doi.org/10.1016/S15740080(04)80006-3

Sabatini, F., Cáceres, G. y Cerda, J. (2001). Segregación residencial en las principales ciudades chilenas: Tendencias de las tres últimas décadas y posibles cursos de acción. EURE, 27(82), 21-42. Recuperado de http://www.eure.cl/index.php/eure/article/view/1258 
Sanz, A. (2005). El viaje de las palabras. Informe de Valladolid, 5(1). 87-93. Recuperado de https://silo.tips/download/el-viaje-de-las-palabras-1

Salazar-Burrows, A., Ugarte, C., y Osses, P. (2014). Exclusión social asociada al transporte y su relación con la distribución de la densidad de población en la provincia de Melipilla, Región Metropolitana de Santiago de Chile. Revista de geografía Norte Grande, 1(59), 145-164. DOI: https://dx.doi.org/10.4067/S0718-34022014000300009

Sen, A. (1982). Utilitarianism and Beyond. Cambridge, Inglaterra: Cambridge University Press.

Sherraden, M. (1991). Assets and the Poor. New York, Estados Unidos: ME Sharpe.

Ssewanyana, S. (2009). Growth, inequality, cash transfers and poverty in Uganda. Country Study, 1(19), 2-28. Recuperado de https://www.econstor.eu/handle/10419/71771

Thang, N., Trung, L.D., Dat, V.H. y Phuong, N.T. (2006). Poverty, poverty reduction and poverty dynamics in Vietnam. Chronic Poverty Research Centre. Recuperado de http://www.chronicpoverty.org/uploads/publication files/CPR2 Background Papers NguyenLe Dang-Vu Hoang.pdf

Thorson Jorgensen, O. Sustentabilidad en la movilidad: vivir con seguridad según las posibilidades colectivas. ACE: Architecture, City and Environment. 7(19). 77-94. DOI: https://doi.org/10.5821/ace.v7i19.2562

Timmins, C. y Murdock, J. (2007). A revealed preference approach to the measurement of congestion in travel cost models. Journal of Environmental Economics and management, 53(2), 230-249. DOI: https://doi.org/10.1016/j.jeem.2006.08.002

Tolley, R. y Turton, B.J. (2014). Transport systems, policy and planning: a geographical approach. New Tork, Estados Unidos: Routledge.

Vakis, R., Rigolini, J. y Lucchetti, L. (2016). Left behind: chronic poverty in Latin America and the Caribbean. DOI: https://doi.org/10.1596/978-1-4648-0660-5

Valencia, E. (2008). Las Transferencias Monetarias Condicionadas Como Política Social en América Latina. Un Balance: Aportes, Límites y Debates. Annual Review of Sociology, 34(1), 499-524. DOI: https://doi.org/10.1146/annurev.soc.052708.074708

Vekemans, R. y Silva, I. (1969). La Marginalidad en América Latina: un ensayo de conceptualización. Santiago de Chile, Chile: DESAL.

Whitelegg, J. (1997). Critical mass: transport, environment and society in the twenty-first century. Londres, Inglaterra: Pluto Press.

Zhao, Z. y Levinson, D. (2012). Introduction to the special issue on value capture for transportation finance. Journal of Transport and Land Use, 5(1), 1-3. DOI: https://doi.org/10.5198/jtlu.v5i1.361

ACE, 16 (47) CC BY-ND 3.0 ES | UPC Barcelona, España ¿QQué variables de movilidad están asociadas con la pobreza 